\title{
Promotion of root elongation by pyridinecarboxylic acids known as novel cut flower care agents
}

\author{
Shigeru Satoh and Yoshihiro Nomura \\ Faculty of Agriculture, Ryukoku University, Otsu 520-2194, Japan. \\ Corresponding author: S. Satoh, E-mail: ssatoh@agr.ryukoku.ac.jp, Phone: +81-77-599-5700, Fax: +81-77-599- \\ 5700
}

Received on December 12, 2016; Accepted on March 30, 2017

\begin{abstract}
Pyridinedicarboxylic acid (PDCA) analogs, including 2,3-, 2,4-, 2,5-, 2,6-, 3,4- and 3,5PDCA, accelerate flower opening and retard senescence of spray-type carnation flowers. In addition the present study revealed that 2,3-PDCA promoted root elongation in lettuce, carrot and rice seedlings, whereas 2,4-PDCA inhibited it. Then, the action of PDCA and pyridinecarboxylic acid (PCA) analogs on root elongation was further examined using rice seedlings. 2,3-, 3,4- and 3,5PDCA promoted rice root elongation, whereas 2,4and 2,6-PDCA inhibited, and 2,5-PDCA had little effect. 3-PCA (nicotinic acid) promoted rice root elongation, but 2- and 4-PCA did not. Moreover, 3-PCA amide (nicotinamide) did not promote root elongation. These findings indicated that a carboxyl group substituted on position 3 of the pyridine ring is necessary to promote root elongation, and that the promoting effect of 3-PCA was not from its action as vitamin $B_{3}$, but from its intrinsic activity as a $3-\mathrm{COOH}$ substituted pyridine. On the other hand, all the PCA and PDCA analogs tested in this study, except 2,6-PDCA and 4-PCA, promoted shoot elongation of rice seedlings.
\end{abstract}

Keywords: 3-pyridinecarboxylic acid, pyridinecarboxylic acids, pyridinedicarboxylic acids, rice seedlings, root elongation

\begin{abstract}
Abbreviations: ACC, 1-aminocyclopropane-1-carboxylic acid; GA, gibberellin; LPB, Light Pink Barbara; PCA, pyridinecarboxylic acid; PDCA, pyridinedicarboxylic acid; PPFD, photosynthetic photon flux density
\end{abstract}

\section{Introduction}

Vlad et al. (2010) reported that 2,4-PDCA inhibited ethylene production in detached flowers of 'White Sim' carnation, which belongs to a standard category of carnation flowers with only one flower per stalk (stem), and delayed senescence of the flowers. This action of 2,4-PDCA was later confirmed with spraytype carnation flowers, such as 'Light Pink Barbara (LPB)', which have a main stalk with several offshoots, each having one or two flowers at their tips (Satoh et al. 2014). Then, Sugiyama and Satoh (2015) revealed that PDCA analogs, including 2,3-, 2,4-, 2,5-, 2,6-, 3,4- and 3,5-PDCA, could accelerate flower bud opening in addition to prolong the vase life of spray-type carnation flowers. Among the PDCA analogs, 2,3-PDCA and 2,4-PDCA were markedly effective in extending the vase life and accelerating flower bud opening in 'LPB' carnation flowers.

Regarding the action mechanism of 2,4-PDCA for retarding senescence of carnation flowers, Vlad et al. (2010) hypothesized that 2,4-PDCA inhibits ACC oxidase by competing with ascorbate, a cosubstrate of the enzyme action. Then, Fragkostefanakis et al. (2013) showed that 2,4PDCA inhibited the in vitro activity of ACC oxidase prepared from tomato pericarp tissues. Satoh et al. (2014) also showed that 2,4-PDCA inhibited ACC oxidase action using a recombinant enzyme produced in Escherichia coli cells from the carnation ACC oxidase gene (DcACO1 cDNA). These observations supported the hypothesis that 2,4PDCA inhibits ACC oxidase action in carnation flowers and reduces ethylene production. This notion is probably applicable to PDCA analogs other than 2,4-PDCA for their mechanism of action to prolong the vase life of the flowers. It will be necessary in the future to test each chemical in terms of whether it can actually inhibit the action of ACC oxidase.

Satoh S, Nomura Y 2017 Promotion of root elongation by pyridinecarboxylic acids known as novel cut flower care agents. Plant Root 11:40-47. doi:10.3117/plantroot.11.40

Copyrights 2017, Plant Root (JSRR), www.plantroot.org 
On the other hand, the action mechanism of PDCAs for accelerating flower bud opening in carnation remains unresolved. Satoh et al. (2014) hypothesized on the association of gibberellin (GA) with the promoting action of 2,4-PDCA on flower bud opening in cut flowers of spray-type carnation. This hypothesis arose from the notion that 2,4PDCA is a structural analog of 2-oxoglutarate, which is a co-substrate for enzymes acting in GA biosynthesis and inactivation, such as GA $3 \beta$-dioxygenase (GA $3 \beta$-hydroxylase), GA-44 dioxygenase, and GA $2 \beta$-dioxygenase (GA $2 \beta$-hydroxylase) (Hedden and Kamiya 1997, Lange et al. 1994a, b, Smith and MacMillan 1984, 1986).

In the present study, we firstly aimed to test whether 2,3-PDCA and 2,4-PDCA have GA-like activity using a bioassay system, in which exogenously applied GA promotes hypocotyl elongation of lettuce seedlings. Unexpectedly, however, we found that 2,3-PDCA promoted root elongation, whereas 2,4-PDCA inhibited it in lettuce, carrot and rice seedlings. This paper shows the details of these findings as well as the results of further analysis of actions of PDCA and PCA analogs on rice seedling growth.

\section{Materials and Methods}

Seeds

Lettuce (Lactuca sativa 'Wayahead') seeds were purchased from Noguchi Seed Co., Hanno, Saitama, Japan. Carrot (Daucus carota subsp. sativus 'Lovely Carrot') seeds were purchased from Nakahara Seed Product Co., Ltd., Fukuoka, Japan. Rice (Oryza sativa 'Hinohikari') seeds were gifts from Professor T. Itani of Ryukoku University (Seta, Otsu, Japan) or Associate Professor S. Morita of Kyoto Prefectural University (Seika, Kyoto, Japan).

Effects of 2,4-PDCA, 2,3-PDCA and $G A_{3}$ on root elongation of lettuce and carrot seedlings

Lettuce: Lettuce seeds were sown in a plastic petri dish on two layers of filter paper wet with ultra pure water $(>18 \mathrm{M} \Omega)$ for 3 days at $23^{\circ} \mathrm{C}$ under continuous light from white fluorescent lamps $\left(140 \mu \mathrm{mol} \mathrm{m}^{-2} \mathrm{~s}^{-1}\right.$ PPFD). Uniformly germinated seeds were used when radicles protruded $1 \mathrm{~mm}$ from seed coats, and placed on solidified Gellan Gum $(1.0 \%, \mathrm{w} / \mathrm{v})$ in a glass test tube $(\Phi 18 \mathrm{~mm} \times 180 \mathrm{~mm})$. The surface of Gellan gel was $10 \mathrm{~cm}$ high from the bottom of the test tube. The Gellan gel contained 2,3-PDCA or 2,4-PDCA at 0 (control), 0.3, $1,3 \mathrm{mM}$, and $\mathrm{GA}_{3}$ at $0.3 \mathrm{mM}$. The concentrations of PDCAs was adjusted by diluting with the stock solutions $(100 \mathrm{mM}, \mathrm{pH} 7$ adjusted with $\mathrm{NaOH}$ ). The germinated seeds were grown at $23^{\circ} \mathrm{C}$ in the light described above for 7 days, and then photographed.

Carrot: Experiments with carrot seedlings were carried out as described for lettuce seedlings.

Effects of PDCA analogs and $G A_{3}$ on the root and shoot elongation of rice seedlings

Rice seeds were firstly immersed in ultra pure water in a glass beaker at $4^{\circ} \mathrm{C}$ in the dark for 1 week, and germinated in a plastic petri dish on two layers of wet filter paper at $23^{\circ} \mathrm{C}$ in the light described above for 3 days. Uniformly germinated seeds were used when coleoptiles and seminal roots protruded $1 \mathrm{~mm}$ from rice husks. Subsequent treatments were carried out as described for lettuce seedlings, but test chemicals were one of six PDCA analogs at 0 (control), 0.3, 1, $3 \mathrm{mM}$, and $\mathrm{GA}_{3}$ at $0.3 \mathrm{mM}$.

In another experiment in which rice seedlings were cultivated hydroponically, germinated rice seeds were grown in test solutions containing PDCA analogs at 0 (control), $0.01,0.03,0.1$ and $0.3 \mathrm{mM}$ as well as $\mathrm{GA}_{3}$ at $0.3 \mathrm{mM}$. Twenty-five germinated seeds were floated on a plastic mesh (pore size 1 $\mathrm{mm}$ ) using a polyurethane float in a transparent plastic box $[9 \mathrm{~cm}$ (width) $\times 9 \mathrm{~cm}$ (length) $\times 8 \mathrm{~cm}$ (height)] containing $300 \mathrm{~mL}$ of test solution, which was made 5 -cm deep with $3 \mathrm{~cm}$-high free space under a lid. Seven days later, the seedlings were photographed as a whole or separately. Then, roots and shoots were detached from each seedling and photographed. The root and shoot (leaf + leaf sheath) lengths of each seedling were measured with a curvemeter (Pen-type Map-meter Concurve 10, Koizumi Sokki Mfg. Co., Ltd. Nagaoka, Japan) on printed photographs. Total root length of each seedling was obtained as the sum of the lengths of a seminal root and all coronal roots. The seedlings developing only seminal roots without coronal roots and those showing markedly inhibited growth due to microbial infection or other reasons, were eliminated and remaining seedlings were aligned according to total root length. Then 15 seedlings in the middle were chosen for measuring root length. Also, shoot length was determined similarly. The root length and shoot length were determined as shown by the average of 15 seedlings \pm SE.

Effects of PCA analogs as compared with some PDCA analogs on the root and shoot elongation of rice seedlings

The procedure was described in the previous section except for the use of PCA analogs (2-PCA, 3-PCA, 4-PCA and 3-PCA amide) and PDCA analogs (2,3- 
PDCA, 2,4-PDCA and 3,4-PDCA) at 0 (control), $0.03,0.1$ and $0.3 \mathrm{mM}$.

\section{Statistical analysis}

Statistical analyses were carried out by Dunnett's multiple range test using an online statistical analysis program, MEPHAS (http:/www.gen-info. osaka-u.ac.jp/testdocs/tomocom/, November 1, 2016).

\section{Chemicals}

All analogs of PCA and PDCA were purchased from Wako Pure Chemical Industries, Ltd., Osaka, Japan, except 3-PCA (nicotinic acid) and $\mathrm{GA}_{3}$, which were from Kishida Chemical Co., Ltd., Osaka and Tokyo Chemical Industry, Co., Ltd, Tokyo, Japan, respectively.

\section{Results}

Effect of 2,3-PDCA, 2,4-PDCA and $\mathrm{GA}_{3}$ on root elongation of lettuce and carrot seedlings
PDCA promoted flower bud opening in cut spraytype carnation flowers like GA. Therefore, we first examined whether 2,4-PDCA has GA-like activity in lettuce hypocotyls whose elongation is promoted by exogenously applied GA. Also we examined the effect of 2,3-PDCA, since this chemical accelerated flower opening and extended the vase life of carnation flowers like 2,4-PDCA (Sugiyama and Satoh 2015). Unexpectedly, 2,3-PDCA and 2,4PDCA had contrasting effects on the elongation of lettuce roots (Fig. 1). 2,3-PDCA at $0.1 \mathrm{mM}$ promoted root elongation, and the degree of promotion increased up to $1 \mathrm{mM}$, then declined slightly at $3 \mathrm{mM}$, whereas, 2,4-PDCA at $0.1-3 \mathrm{mM}$ severely inhibited the elongation of lettuce roots. $\mathrm{GA}_{3}$ at $0.3 \mathrm{mM}$ promoted hypocotyl elongation but inhibited root elongation in lettuce seedlings.

Carrot: 2,3-PDCA and 2,4-PDCA had similar effects on root elongation of carrot seedlings (Fig. 2). 2,3-PDCA at $0.3-3 \mathrm{mM}$ promoted the elongation of carrot roots, whereas 2,4-PDCA at $0.1-3 \mathrm{mM}$ inhibited it greatly. In addition, 2,4-PDCA inhibited the shoot elongation in carrot seedlings. $\mathrm{GA}_{3}$ at 0.3 $\mathrm{mM}$ inhibited the elongation of carrot roots.

Lettuce: Satoh et al. (2014) hypothesized that 2,4-

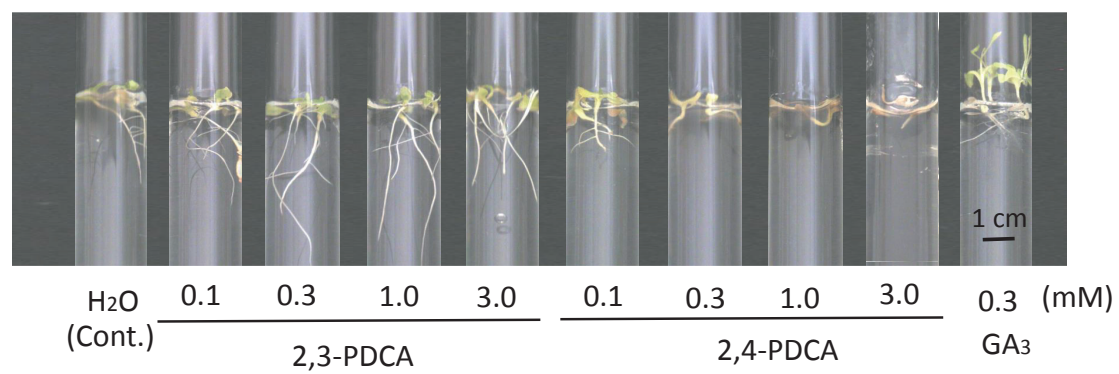

Fig. 1. Effects of 2,3-PDCA, 2,4-PDCA and $\mathrm{GA}_{3}$ in root elongation of lettuce seedlings. Three germinated lettuce seeds with radicles protruded $1 \mathrm{~mm}$ were placed on the surface of solidified Gellan Gum $(0.1 \%$, w/v) containing 2,3-PDCA and 2,4PDCA at $0.1-3 \mathrm{mM}$ and $\mathrm{GA}_{3}$ at $0.3 \mathrm{mM}$ and allowed to grow for 7 days at $23^{\circ} \mathrm{C}$ under light from white fluorescent lamps. Water was used as the control.

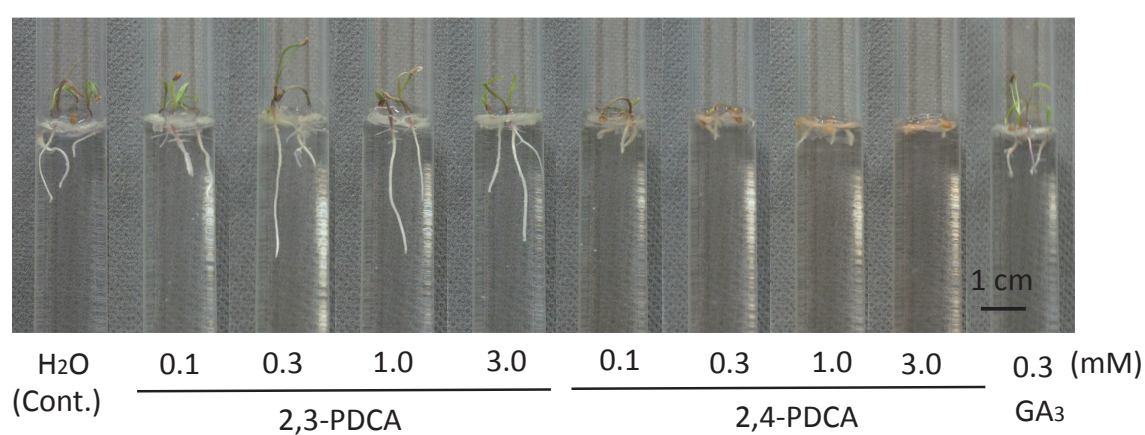

Fig. 2. Effects of 2,3-PDCA, 2,4-PDCA and $\mathrm{GA}_{3}$ on the root elongation of carrot seedlings. Three germinated carrot seeds with radicle protruded to $1 \mathrm{~mm}$ were treated as described in the legend to Fig. 1. 
Effects of PDCA analogs and $\mathrm{GA}_{3}$ on the root and shoot elongation of rice seedlings

The effects of each of six PDCA analogs, 2,3-, 2,4-, 2,5-, 2,6-, 3,4- and 3,5-PDCA, on root growth were further examined using rice seedlings (Fig. 3). 2,3PDCA promoted root elongation and the maximum promotion was observed at $1 \mathrm{mM}$. 3,4-PDCA and 3,5-PDCA also promoted the root elongation, although the latter was effective only at $3 \mathrm{mM}$. On the other hand, 2,4-PDCA at $0.1-3 \mathrm{mM}$ markedly inhibited the root elongation. 2,5-PDCA and 2,6PDCA also inhibited root elongation, but 2,5-PDCA was effective only at 1 and $3 \mathrm{mM} . \mathrm{GA}_{3}$ at $0.3 \mathrm{mM}$ promoted the elongation of shoot (leaf + leaf sheath), but inhibited the elongation of roots.

Figure $4 \mathrm{~A}$ and $\mathrm{B}$ show the effects of PDCA analogs on the root and shoot elongation in rice seedlings. In this experiment, the concentrations of chemicals used were changed to $0.01-0.3 \mathrm{mM}$. 2,3PDCA significantly promoted root elongation at 0.3 $\mathrm{mM}$, but 3,4- and 3,5-PDCAs promoted significantly at 0.1 and $0.3 \mathrm{mM}$, indicating that these chemicals were more effective than 2,3-PDCA. 2,5-PDCA up to $0.3 \mathrm{mM}$ did not affect the root growth of rice seedlings, 2,4-PDCA and 2,6-PDCA at $0.1 \mathrm{mM}$ significantly inhibited the root growth, and the latter seemed to be more inhibitory than the former. $\mathrm{GA}_{3}$ at 0.1 and $0.3 \mathrm{mM}$ significantly inhibited the elongation of rice roots.

On the other hand, shoot elongation of rice seedlings was promoted by all the PDCA analogs, except 2,6-PDCA, at 0.1 and $0.3 \mathrm{mM}$. Although 2,4PDCA promoted it even at $0.03 \mathrm{mM}$, the difference among these chemicals was small. $\mathrm{GA}_{3}$ at $0.01 \mathrm{mM}$ markedly promoted the elongation of rice seedlings (53.2 $\mathrm{mm}$, data not shown). Only 2,6-PDCA inhibited the elongation of rice shoots, but we did not investigate this inhibition further.

Effects of PCA analogs as compared with some $P D C A$ analogs on the root and shoot elongation of rice seedlings

The results shown in the previous section (Figs. 3 and 4) suggested that the carboxyl group at position 3 of the pyridine ring is necessary to promote root elongation in rice seedlings, and probably in lettuce and carrot seedlings. Therefore, we tested the activity of PCA analogs, i.e., 2-, 3- and 4-PCA and

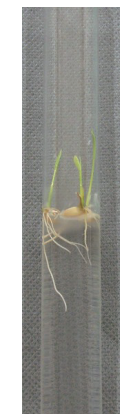

$\mathrm{H}_{2} \mathrm{O}$

Control

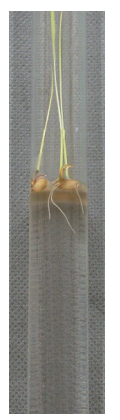

0.3

$\mathrm{GA} 3$
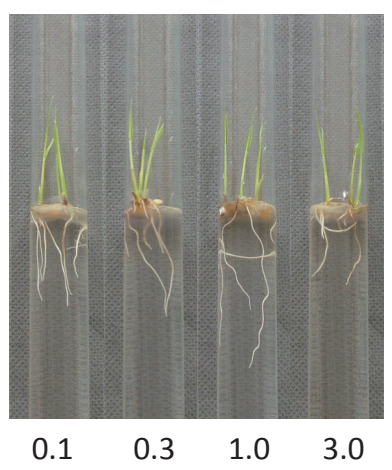

2,3-PDCA
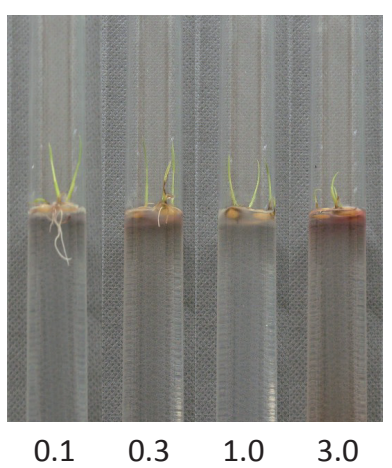

2,6-PDCA

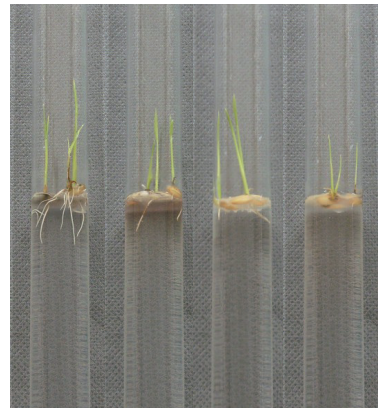

$\begin{array}{llll}0.1 & 0.3 & 1.0 & 3.0\end{array}$

2,4-PDCA

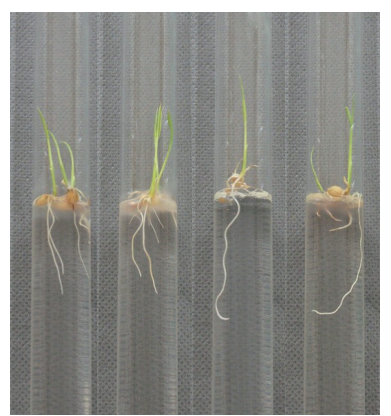

$\begin{array}{llll}0.1 & 0.3 & 1.0 & 3.0\end{array}$ 3,4-PDCA
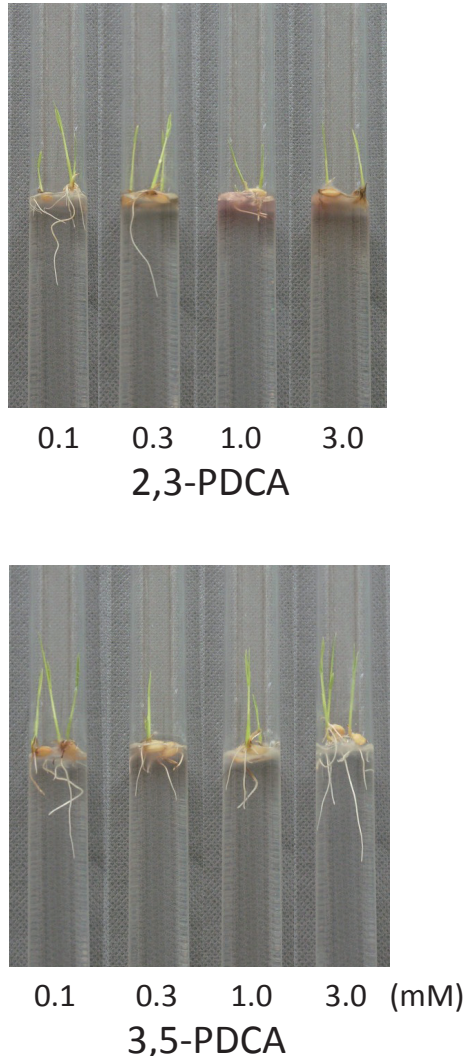

Fig. 3. Effects of 2,3-, 2,4-, 2,5-, 2,6-, 3,4- and 3,5-PDCA and $\mathrm{GA}_{3}$ on root elongation of rice seedlings. Three germinated rice seeds with both seminal root and coleoptile protruded about $1 \mathrm{~mm}$ were treated similarly as described in the legend to Fig. 1. Root system was mainly consisted of a seminal root when photographed. 
A.

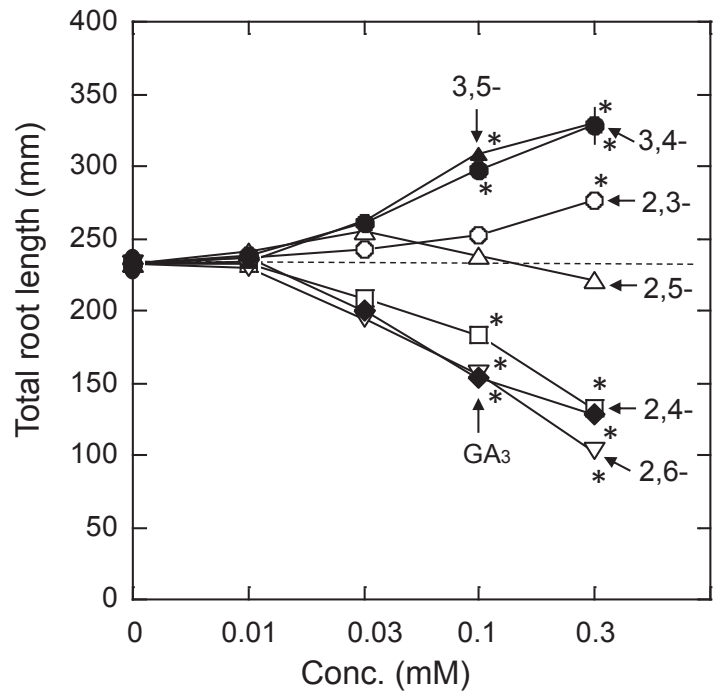

B.

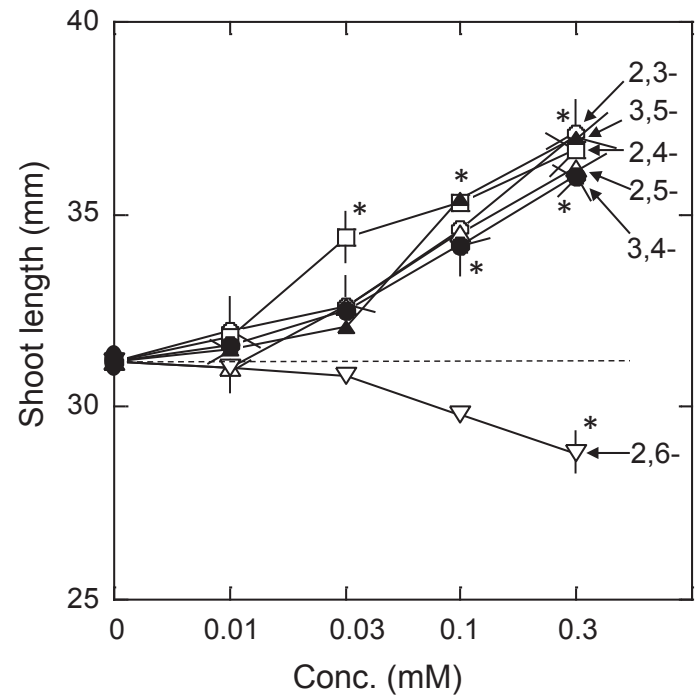

Fig. 4. Comparison among PDCA analogs (2,3-, 2,4-, 2,5-, 2,6-, 3,4- and 3,5-PDCA) and $\mathrm{GA}_{3}$ on their effects on root elongation (A) and shoot elongation (B) in rice seedlings. Germinated rice seeds were grown hydroponically at $23^{\circ} \mathrm{C}$ for 7 days in test solutions containing respective PDCAs and $\mathrm{GA}_{3}$ at 0 (control), 0.01, 0.03, 0.1 and $0.3 \mathrm{mM}$. Root system was consisted of a seminal root and several coronal roots. Total root length per seedling was determined by the sum of length of seminal and coronal roots. Total root length was determined as the mean of 15 seedlings in the middle range of 25 seedlings without showing an abnormal growth. Data are shown by the mean \pm SE of 15 seedlings, but SEs are shown by vertical or diagonal lines when they are larger than the size of symbols. $\mathrm{GA}_{3}$ at $0.01 \mathrm{mM}$ markedly promoted the elongation of rice seedling, attaining $53.2 \mathrm{~mm}$, but data are not shown. * shows significant difference from the control by Dunnett's multiple range test $(P<0.05)$, and symbols between top and bottom stars at given concentrations are all significantly different from the control.

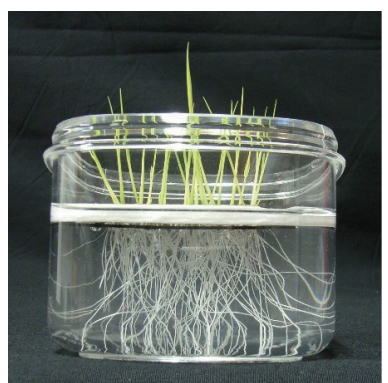

Control (H2O)

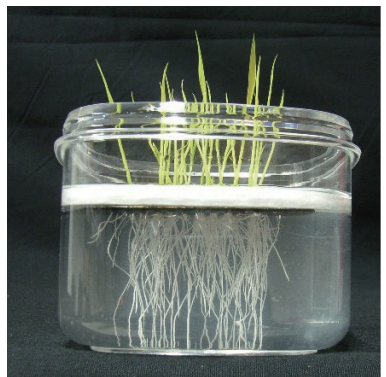

3-PCA amide

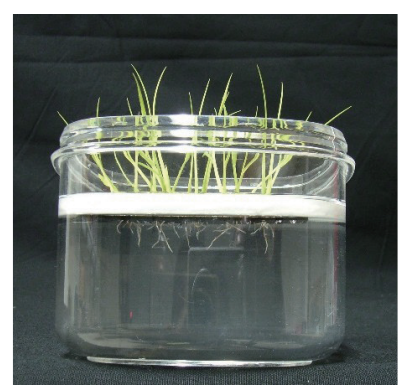

2-PCA

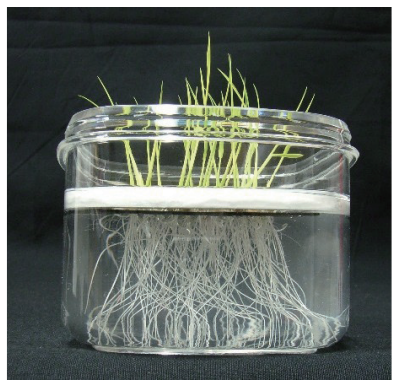

2,3-PDCA

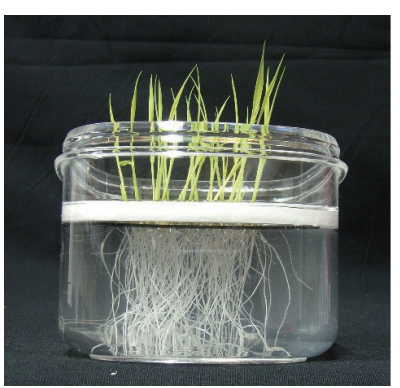

3-PCA

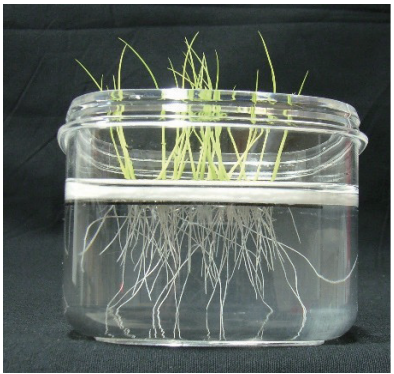

2,4-PDCA

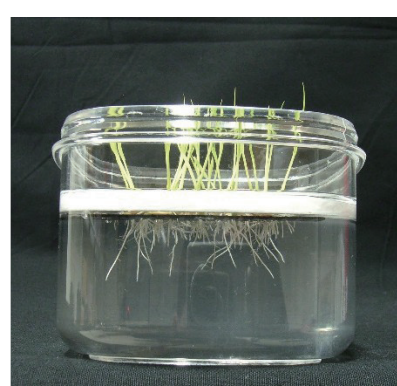

4-PCA

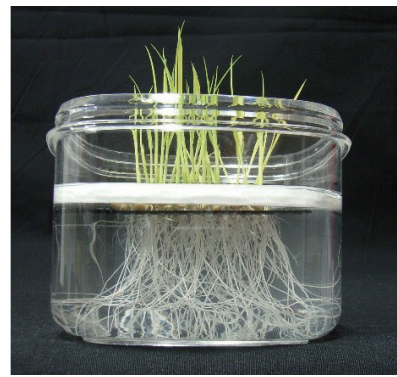

3,4-PDCA

Fig. 5. Effects of PCA analogs (2-, 3-, 4-PCA and 3-PCA amide) as compared with those of PDCA analogs (2,3-, 2,4- and 3,4$\mathrm{PDCA}$ ) on growth of rice seedlings. Germinated rice seeds were grown at $23^{\circ} \mathrm{C}$ for 7 days hydroponically in test solutions containing each chemical at $0.3 \mathrm{mM}$. 
A.

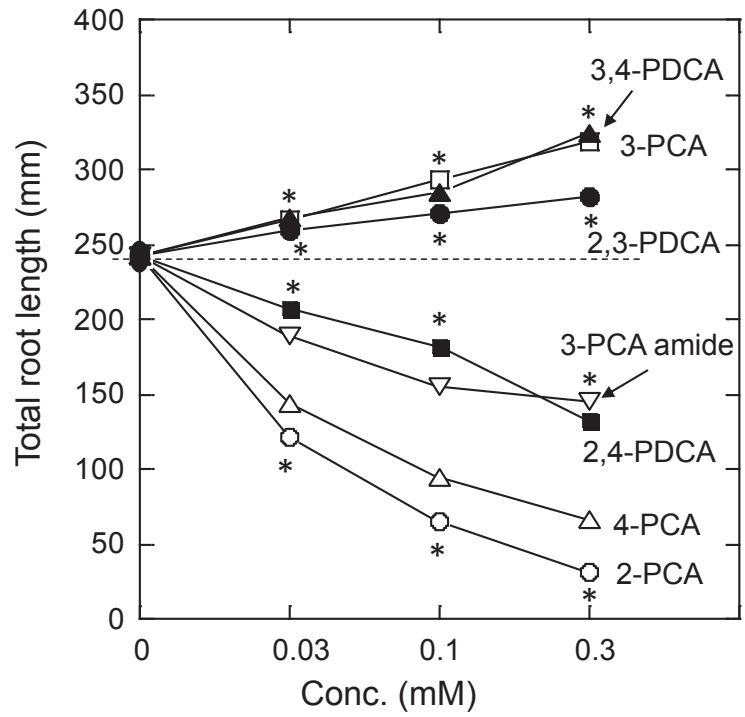

B.

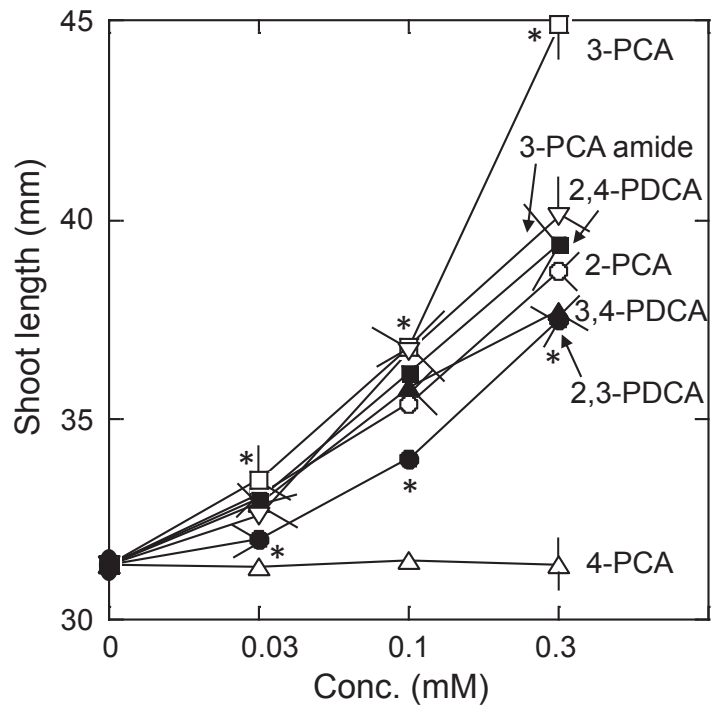

Fig. 6. Quantitative analysis of the effects of PCA analogs (2-, 3-, 4-PCA and 3-PCA amide) as compared with those of PDCA analogs (2,3-, 2,4- and 3,4-PDCA) on the root elongation (A) and shoot elongation (B) of rice seedlings. Concentrations of each chemical were 0 (control), 0.03, 0.1 and $0.3 \mathrm{mM}$. Rice seedlings obtained in the previous experiment shown in Fig. 5 were used to determine the average total root length and shoot length as described in the legend to Fig. 4. Statistical analysis and data presentation are similar to that described in the legend to Fig. 4.

3-PCA amide, as well as 2,3-, 2,4- and 3,4-PDCA, on the growth of rice seedlings grown for 7 days by hydroponic culture. Figure 5 shows the seedlings treated with these chemicals at $0.3 \mathrm{mM}$. The apparent mass of roots (both seminal and coronal roots) was clearly increased by 3-PCA, 3,4-PDCA, and 2,3-PDCA, but decreased by 2-PCA, 4-PCA and 2,4-PDCA. Interestingly, 3-PCA amide decreased the mass of roots, although not as much as 2-PCA, 4-PCA and 2,4-PDCA. On the other hand, all the chemicals, except 4-PCA, appeared to promote the elongation of shoots, which was judged from the protrusion of shoot over the edge of containers.

Figure $6 \mathrm{~A}$ and $\mathrm{B}$ show the total length of root and shoot per seedling, respectively, treated with PCA and PDCA analogs at $0.03-0.3 \mathrm{mM}$. Root elongation was significantly promoted by 3 -PCA, 3,4-PDCA and 2,3-PDCA at all the concentrations used. The magnitude of promotion was similar in 3-PCA and 3,4-PDCA, followed by 2,3-PDCA. However, 2-PCA, 4-PCA, 2,4-PDCA, and 3-PCA amide significantly inhibited the root elongation at all the concentrations used. 2-PCA had the greatest inhibitory effect on root elongation, followed by 4-PCA, 2,4-PDCA and 3-PCA amide. On the other hand, all the chemicals, except 4-PCA, promoted significantly the elongation of shoots of rice seedlings. 3-PCA was most active in the promotion of shoot elongation in rice seedlings, followed by
3-PCA amide, 2,4-PDCA and 2-PCA. 4-PCA did not affect the shoot elongation of rice seedlings.

\section{Discussion}

At first, we intended to investigate whether PDCA analogs, such as 2,4-PDCA and 2,3-PDCA, have GA-like activity by examining their effects on lettuce hypocotyl elongation, a well-known bioassay system for GA. At the same time we examined the effect of $\mathrm{GA}_{3}$ at $0.3 \mathrm{mM}$ as a positive reference. This concentration of $\mathrm{GA}_{3}$ was used to fall in the range of concentrations of PDCA analogs, which was preliminarily determined to be effective using 2,4- and 2,3-PDCA (Data not shown). $\mathrm{GA}_{3}$ at 0.3 $\mathrm{mM}$ actually promoted the hypocotyl elongation of lettuce seedlings (Fig. 1), whereas, the root elongation was partly inhibited by $\mathrm{GA}_{3}$ at $0.3 \mathrm{mM}$. Inhibition of root elongation by $\mathrm{GA}_{3}$ at $0.3 \mathrm{mM}$ was further confirmed with carrot and rice seedlings (Figs. 2-4). Previously, Tanimoto (1987) reported that $\mathrm{GA}_{3}$ at $10 \mathrm{nM}$ (a determined optimum concentration) promoted root elongation of lettuce seedlings only when it was supplied with inhibitors of GA biosynthesis. The findings suggest that GA content is saturated in lettuce root and exogenous application of $\mathrm{GA}_{3}$ does not promote elongation of the roots. Inhibition of root elongation in the present study was probably caused by an extraordinary high concentration of applied $\mathrm{GA}_{3}$. On the other hand, 
2,4-PDCA and 2,3-PDCA at up to $3 \mathrm{mM}$ did not promote the elongation of lettuce hypocotyls (Fig. 1). These observations suggest little if any association of GA in the action of PDCA analogs. Therefore, we did not further discuss the association of GA in the action of PDCA, and rather concentrated on the difference among PDCA and PCA analogs in the inhibition of root elongation as shown below.

PDCA analogs were previously shown to accelerate flower bud opening and retard senescence of spray-type carnation flowers (Sugiyama and Satoh 2015). Among the PDCA analogs, 2,3-PDCA and 2,4-PDCA had marked activity and were thought to be suitable agents for treatment of the flowers. Interestingly, the present study revealed that they had opposite effect on the root elongation of lettuce, carrot and rice seedlings; 2,3-PDCA promoted, whereas 2,4-PDCA inhibited root elongation (Figs. 1-3). On the other hand, both PDCA analogs promoted the elongation of shoot (leaf + leaf sheath) in rice seedlings (Figs. 4-6).

Precise analyses of the effects of PDCA analogs and PCA analogs revealed that acceleration of root elongation in rice seedlings depends on the free carboxyl group $(-\mathrm{COOH})$ at position 3 of the pyridine ring, and the inhibition of root elongation depends on $-\mathrm{COOH}$ at positions 2 or 4 of the ring (Figs. 3-6). When the second carboxyl group was introduced into 3-PCA, its introduction to position 2 of the pyridine ring, which makes 2,3-PDCA, reduced the promoting activity of 3-PCA more severely than that into position 4 of the pyridine ring, which makes 3,4-PDCA (Figs. 5 and 6). Moreover, in PDCAs, 3-COOH overcame the inhibitory effects of 2- or 4-COOH; in other words, the latter two - $\mathrm{COOHs}$ could not nullify the accelerative effect of 3-COOH.

Interestingly, when 3-COOH of 3-PCA was replaced with $-\mathrm{CONH}_{2}$, the resultant 3-PCA amide (nicotinamide) lost the root elongation promoting activity in rice seedlings (Fig. 5). Both 3-PCA and 3-PCA amide are vitamin $\mathrm{B}_{3}$, known as nicotinic acid and nicotinamide, respectively, and are regarded to have activity equivalent to the vitamin. The present findings that 3-PCA promoted but 3-PCA amide inhibited rice root elongation suggested that the root elongation promoting activity of 3-PCA did not originate from its activity as vitamin $\mathrm{B}_{3}$.

The present findings showed the promoting effects of 3-PCA and PDCA analogs with 3-COOH on root elongation in rice seedlings. This notion may also apply to the promotion of root elongation in lettuce and carrot seedlings.
On the other hand, all the PCA and PDCA analogs, except 4-PCA and 2,6-PDCA, tested in the present study promoted the shoot elongation of rice seedlings. However, the mechanism of these diverse effects of PCA and PDCA analogs remain unknown. Previously, 2,4-PDCA was shown to inhibit the activity of prolyl-4-hydroxylase by acting as a structural analog of 2-oxoglutarate, the co-substrate of the enzyme (Vlad et al. 2010), and that of ACC oxidase by acting as a possible structural analog of ascorbate, the co-substrate of the enzyme (Vlad et al. 2010, Satoh et al. 2014). Moreover, aconitase has been reported to be inactivated markedly by $2,6-$ PDCA, but not by 2,3-, 2,4-, and 2,5-PDCA (Murakami et al. 2007). However, at present, it is uncertain whether these enzymes are the primary target of PCA and PDCA action in the modification of root elongation. The mechanism of action of PCA and PDCA analogs on root growth will hopefully be elucidated in the near future.

Promotion of root elongation in the seedling of vegetable and ornamental crops by PDCA and PCA analogs probably has merits in practical agriculture, since massive roots of seedlings surely promote root establishment of the seedlings after transplanting. From this point of view, we are now investigating a practical method to apply these chemicals to grow sound and vigorous seedlings of vegetable and ornamental crops.

\section{Acknowledgements}

This study was supported financially by a Matching Planner Program Grant (MP27115663028 to S. Satoh) from the Japan Science and Technology Agency and a Grant-in-Aid (16K07604 to S. Satoh) for Scientific Research from the Japan Society for the Promotion of Science.

\section{References}

Fragkostefanakis S, Kalaitzis P, Siomos AS, Gerasopoulos D 2013 Pyridine 2,4-dicarboxylate downregulated ethylene production in response to mechanical wounding in excised mature green tomato pericarp discs. J. Plant Growth Regul. 32: 140-147.

Hedden P, Kamiya Y 1997 Gibberellin biosynthesis: enzymes, genes and their regulation. Annu. Rev. Plant Physiol. Plant Mol. Biol. 48: 431-460.

Lange T, Hedden P, Graebe JE 1994a Expression cloning of a gibberellin 20-oxidase, a multifunctional enzyme involved in gibberellin biosynthesis. Proc. Natl. Acad. Sci. USA 91: 8552-8556.

Lange T, Schweimer A, Ward DA, Hedden P, Graebe JE 1994b Separation and characterization of three 2-oxoglutarate-dependent dioxygenases from Cucurbita maxima L. endosperm involved in gibberellin 
biosynthesis. Planta 195: 98-107.

Murakami K, Haneda M, Hosokawa Y, Yoshino M 2007 Prooxidant action of pyridine carboxylic acids: transition metal-dependent generation of reactive oxygen species. Trace Nut. Res. 24: 49-55.

Satoh S, Kosugi Y, Sugiyama S, Ohira I 2014 2,4Pyridinedicarboxylic acid prolongs the vase life of cut flowers of spray carnations. J. Japan. Soc. Hort. Sci. 83: 72-80.

Smith VA, MacMillan J 1984 Purification and partial characterization of a gibberellin $2 \beta$-hydroxylase from Phaseolus vulgaris. J. Plant Growth Regul. 2: 251-264.

Smith VA, MacMillan J 1986 The partial purification and characterisation of gibberellin $2 \beta$-hydroxylases from seeds of Pisum sativum. Planta 167: 9-19.

Sugiyama S, Satoh S 2015 Pyridinedicarboxylic acids prolong the vase life of cut carnation flowers of spraytype 'Light Pink Barbara' carnation by accelerating flower opening in addition to an already-known action of retarding senescence. Hort J. 84: 172-177.

Tanimoto E 1987 Gibberellin-dependent root elongation in Lactuca sativa: Recovery from growth retardantsuppressed elongation with thickening by low concentration of $\mathrm{GA}_{3}$. Plant Cell Physiol. 28: 963-973.

Vlad F, Tiainen P, Owen C, Spano T, Daher FB, Oualid F, Senol NO, Vlad D, Myllyharju J, Kalaitzis P 2010 Characterization of two carnation petal prolyl 4 hydroxylases. Physiol. Plant. 140: 199-207. 